\title{
Impact of obesity in colorectal endoscopic submucosal dissection: single-center retrospective cohort study
}

\author{
Jun Tachikawa ${ }^{1,2}$, Hideyuki Chiba', Naoya Okada1', Jun Arimoto', Keiichi Ashikari², Hiroki Kuwabara', \\ Michiko Nakaoka', Takuma Higurashi ${ }^{2}$, Toru Goto ${ }^{1}$ and Atsushi Nakajima2*
}

\begin{abstract}
Background: When performing colorectal endoscopic submucosal dissection (ESD) in obese patients, technically difficult cases are sometimes experienced because of difficulty with the insertion of the colonoscope, poor scope maneuverability, or an abundance of fat tissue in the submucosal layer. Since the association between obesity and colorectal ESD has not been investigated, we evaluated the clinical impact of obesity in patients who underwent colorectal ESD.
\end{abstract}

Methods: We retrospectively reviewed 535 patients who underwent colorectal ESD between April 2012 and February 2019. Patients were divided into three groups based on their body mass index (BMl): a control group (BMl $<25 \mathrm{~kg} /$ $\left.\mathrm{m}^{2}\right)$, an overweight group $\left(25 \mathrm{~kg} / \mathrm{m}^{2} \leq \mathrm{BMl}<30 \mathrm{~kg} / \mathrm{m}^{2}\right)$, and an obese group $\left(\mathrm{BMI} \geq 30 \mathrm{~kg} / \mathrm{m}^{2}\right)$, and the short-term clinical outcomes were analyzed to assess the safety and difficulty of colorectal ESD.

Results: No significant difference in the procedure times, en bloc resection rates, pathological diagnoses, or complications were seen among the groups. While the amount of sedative per body weight was significantly lower in the group with a higher BMI (flunitrazepam: $1.75 \times 10^{-2}\left[1.27 \times 10^{-2}-2.34 \times 10^{-2}\right] \mathrm{mg} / \mathrm{kg}$ vs. $1.48 \times 10^{-2}\left[1.08 \times 10^{-2}-\right.$ $\left.2.03 \times 10^{-2}\right] \mathrm{mg} / \mathrm{kg}$ vs. $1.16 \times 10^{-2}\left[0.98 \times 10^{-2}-1.54 \times 10^{-2}\right] \mathrm{mg} / \mathrm{kg}, P<0.001 ;$ pethidine: 0.63 [0.55-0.72] mg/kg vs. $0.50[0.46-0.56] \mathrm{mg} / \mathrm{kg}$ vs. 0.39 [0.32-0.45] mg/kg, $P<0.001)$, a reduction in percutaneous arterial oxygen saturation occurred significantly more frequently in the group with a higher BMI (123 [30.2\%] vs. 43 [43.9\%] vs. 10 [55.6\%], $P=0.005)$. When the procedures were performed by trainees, the number of cases that required a procedure time of longer than 90 min was significantly larger in the group with a higher BMI (27 [10.8\%] vs. 14 [21.9\%] vs. 3 [25.0\%], $P=0.033)$.

Conclusions: This study showed that colorectal ESD could be performed safely and effectively in obese patients. However, ESD in obese patients requires attention, particularly to changes in respiratory conditions.

Keywords: Colorectal cancer, Endoscopic submucosal dissection, Obesity, Body mass index

*Correspondence: nakajima-tky@umin.ac.jp

2 Department of Gastroenterology and Hepatology, Yokohama City University Graduate School of Medicine, 3-9, Fukuura, Kanazawa-Ku, Yokohama 236-0004, Japan

Full list of author information is available at the end of the article

\section{Background}

The number of obese people is increasing worldwide and obesity has been shown to have various effects on the clinical outcomes of a variety of diseases. Several studies have reported an association between obesity and surgery, suggesting that the risks of peri- and postoperative complications associated with surgery for colorectal cancer are higher for obese patients [1-6]. In 
obese patients, securing an operative field during surgery can be difficult because of a thick abdominal wall and abundance of visceral fat. In addition, since fat is a very poor conductor of electricity, unnecessary invasion to other organs is likely to occur. Therefore, obesity has been associated with longer operation times, longer hospital stays, and postoperative complications such as cardiovascular disease, pulmonary embolism, and wound infections. However, a significant difference in mortality has not been observed.

On the other hand, colorectal endoscopic submucosal dissection (ESD) has become widespread as a treatment for early colorectal cancer. Although ESD for large lesions has become possible, mainly in Japan and Asia [7], such procedures remain challenging in Western countries. Several reports have described the difficulties of treating such lesions, including fibrosis and poor scope manipulation $[8,9]$, but no reports have examined the relationship between ESD and obesity. According to a Korean study, gastric ESD in obese patients was associated with a longer procedure time; however, no differences in the complication rates and en bloc resection rates were seen [10]. In that study, fatty tissue in the submucosal layer was suggested to have complicated the ESD procedures. When abundant fat tissue is present in the submucosal layer, securing a field of view can be difficult, and the submucosal tissue is more likely to be burnt, potentially resulting in poor cauterization against bleeding (Fig. 1) $[11,12]$. In addition, when performing colorectal ESD in obese patients, technically difficult cases are sometimes experienced because of difficulty with the insertion of the colonoscope or poor scope maneuverability. However, the impact of obesity on the outcomes of patients undergoing colorectal ESD has not yet been directly assessed. In this study, we clarified the clinical impact of obesity on the short-term outcomes of ESD for colorectal neoplastic lesions not only during hospitalization, but also with respect to delayed events after ESD.

\section{Methods \\ Patients}

We retrospectively reviewed 535 patients who underwent colorectal ESD between April 2012 and February 2019 at our hospital. Patients whose body mass index (BMI) was unknown, in whom ESD was discontinued, and who presented with neuroendocrine tumors were excluded. The remaining 523 patients were divided into three groups based upon their BMI according to the World Health Organization's criteria for obesity: control group $\left(\mathrm{BMI}<25 \mathrm{~kg} / \mathrm{m}^{2}\right)$, overweight group $(25 \mathrm{~kg} /$ $\left.\mathrm{m}^{2} \leq \mathrm{BMI}<30 \mathrm{~kg} / \mathrm{m}^{2}\right)$, and obese group $\left(\mathrm{BMI} \geq 30 \mathrm{~kg} / \mathrm{m}^{2}\right)$ [13]. Informed consent was obtained from each patient included in the study.

\section{ESD Procedures}

The ESD procedure were performed using a single-channel endoscope (PCF-Q260 JI, GIF-Q260J; Olympus Co., Tokyo, Japan) with a transparent attachment (D-20111802; Olympus Co.), and carbon dioxide was used for insufflation. The tumor resection devices were the FlexKnife $^{\circledR}$ (KD-630L; Olympus Co.) or the DualKnife ${ }^{\circledR}$ (KD-650L; Olympus Co.) with a VIO 300D high-frequency generator (ERBE, Tübingen, Germany), and the solution for local injection was a mixture of $0.4 \%$ sodium hyaluronate and indigo carmine with diluted epinephrine. In all the cases, patients were dosed with $2 \mathrm{~L}$ of polyethylene glycol electrolyte solution to prepare the bowel prior to ESD. For conscious sedation, flunitrazepam (0.2$0.5 \mathrm{mg}$ ) and pethidine (17.5-35 mg) were administered for conscious sedation at the beginning of the procedure and added as appropriate. Glucagon or scopolamine was injected intravenously to inhibit peristalsis of the colon
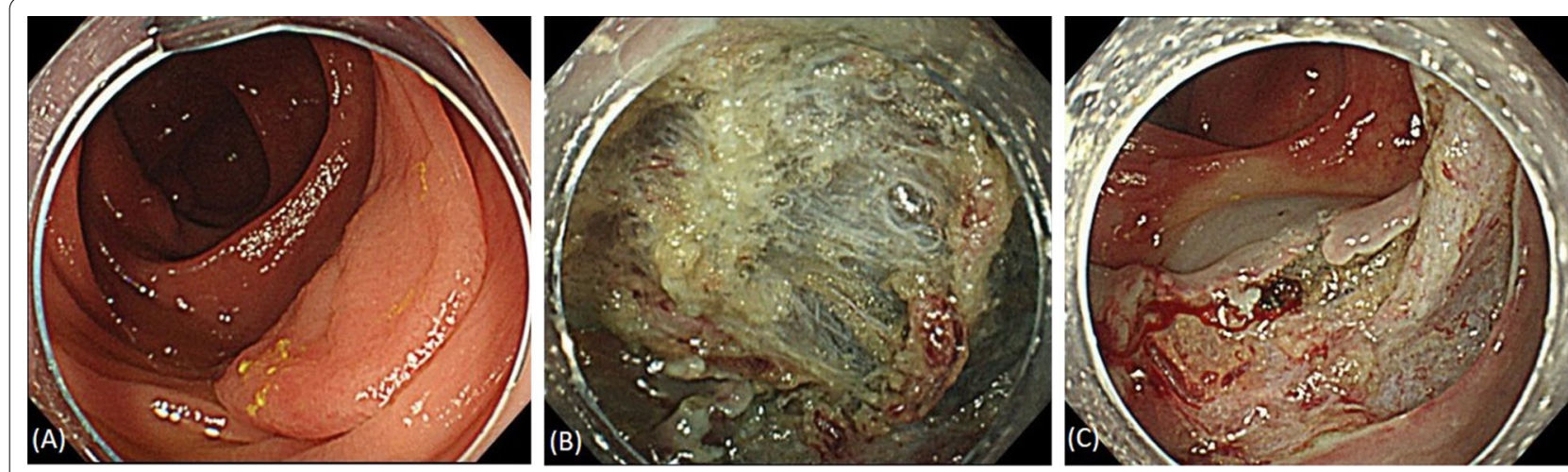

Fig. 1 Lesion with abundant fat tissue in the submucosal layer. A Non-granular laterally spreading tumor that was $34 \mathrm{~mm}$ in size and located in the ascending colon of an obese patient whose body mass index was $26.7 \mathrm{~kg} / \mathrm{m}^{2}$. B Abundant fat tissue in the submucosa. C Fat tissue which was also found on the ulcer after resection 
during the procedure. Blood pressure, heart rate, electrocardiography, and oxygen saturation were monitored during the procedure. The procedures were performed by one expert who had performed $>200$ colorectal ESD procedures (as of 2012) and 6 trainees, each of who had performed $>30$ gastric ESD procedures and $<100$ colorectal ESD procedures. The expert was always present to supervise and guide the ESD procedures. The expert took over the operation from the trainee when any of the following conditions were met: (1) the total procedure time exceeded about $2 \mathrm{~h}$; (2) perforation occurred or was likely to have occurred; (3) continuation of the procedure seemed difficult; or (4) a better resection method needed to be taught. The mucosal incision was made using the End-cut I mode (effect 2, duration 2, interval 2), and the submucosal dissection was performed primarily in the End-cut I mode and sometimes in swift coagulation mode (effect 2, $45 \mathrm{~W}$ ) for submucosal areas rich in vessels or fat tissue [9].

\section{Definitions and outcome measurements}

The ESD procedure time was defined as the elapsed time from the start of the local injection until the complete removal of the lesion. Based on previous reports, we defined a "prolonged procedure" as one that required more than 90 min [14-16]. The degree of submucosal fibrosis was defined according to previous reports as follows: F0, no fibrosis, manifesting as a blue transparent layer; F1, mild fibrosis, appearing as a white web-like structure in the blue submucosal layer; and F2, severe fibrosis, appearing as a white muscular structure without a blue transparent layer in the submucosal layer [17]. The specimen size was defined as the major diameter of the resected sample. Systolic blood pressure fluctuations, bradycardia, and hypoxemia were defined as occurring if there was a change in blood pressure, heart rate, or percutaneous arterial oxygen saturation $\left(\mathrm{SpO}_{2}\right)$ at any point during treatment. We defined delayed bleeding after ESD as a decrease in the hemoglobin level by at least $2 \mathrm{~g} / \mathrm{dL}$ or more below the most recent preoperative level, and/ or the necessitation of blood transfusion and/or marked hematochezia $[18,19]$. We defined a delayed event after colorectal ESD as an adverse event other than bleeding and perforation if it occurred within 30 days after ESD was performed.

\section{Histopathological assessment}

All specimens were cut into $2 \mathrm{~mm}$ slices and stained with hematoxylin and eosin. The specimens were examined by experienced pathologists to determine the histological type, depth of invasion, the presence/absence of lymphatic invasion and vascular involvement, and the lateral and vertical resection margins. En bloc resection was defined as the removal of the tumor in a single piece, and complete resection was defined as en bloc resection with vertical and horizontal margin negative. Patients were defined as having undergone curative resection when the following criteria based on the Japanese Classification for Cancer of the Colon and Rectum were met: lateral and vertical margins were free of tumor cells, the tumor was an intramucosal carcinoma or a carcinoma with mild submucosal invasion (invasion depth of less than $1000 \mu \mathrm{m}$ ), and lymphatic invasion, vascular involvement, grade 2 or 3 tumor budding, or a poorly differentiated tumor component were absent [20].

\section{Statistical analyses}

All the variables were tested for normality using the Shapiro-Wilk test; nonparametric tests were used for data that were not normally distributed. Continuous data were presented as the median and interquartile ranges, and categorical data were presented as quantities and proportions. For the statistical analyses, we used a chi-squared test and a Kruskal-Wallis test. All the analyses were performed with SPSS 23 for Windows. $P$ values $\leq 0.05$ were considered to denote statistical significance.

\section{Ethics}

The study was conducted in accordance with the principles laid down in the Declaration of Helsinki, and with the approval of the Institutional Review Board of Omori Red Cross Hospital (No. 19-31).

\section{Results}

\section{Baseline characteristics}

A total of 535 patients who received ESD for a colon tumor during the study period were evaluated. The total number of lesions was 598. Patients who were treated for a neuroendocrine tumor $(n=7)$, did not have BMI records $(n=2)$ or whose ESD was discontinued $(n=3)$ were excluded. The remaining 523 patients (586 lesions) were analyzed in our study (Fig. 2). The baseline characteristics of the patients and their lesions are listed in Table 1 . The median age (interquartile range) was 70.0 (62.5-77.0) years, males comprised $57.4 \%(\mathrm{n}=300)$, and the median BMI was $22.8(20.3-24.8) \mathrm{kg} / \mathrm{m}^{2}$. Patients whose BMI was less than $25 \mathrm{~kg} / \mathrm{m}^{2}$ were classified as belonging to the control group, those with a BMI of $25 \mathrm{~kg} / \mathrm{m}^{2}$ or more and less than $30 \mathrm{~kg} / \mathrm{m}^{2}$ were classified as belonging to the overweight groups, and those with a BMI of $30 \mathrm{~kg} / \mathrm{m}^{2}$ or more were classified as belonging to the obese group [13]. No significant difference in median age or the proportion of males: females was seen among the groups. Furthermore, no significant differences in the number of patients with comorbidities and taking antithrombotic medication were seen. No significant 


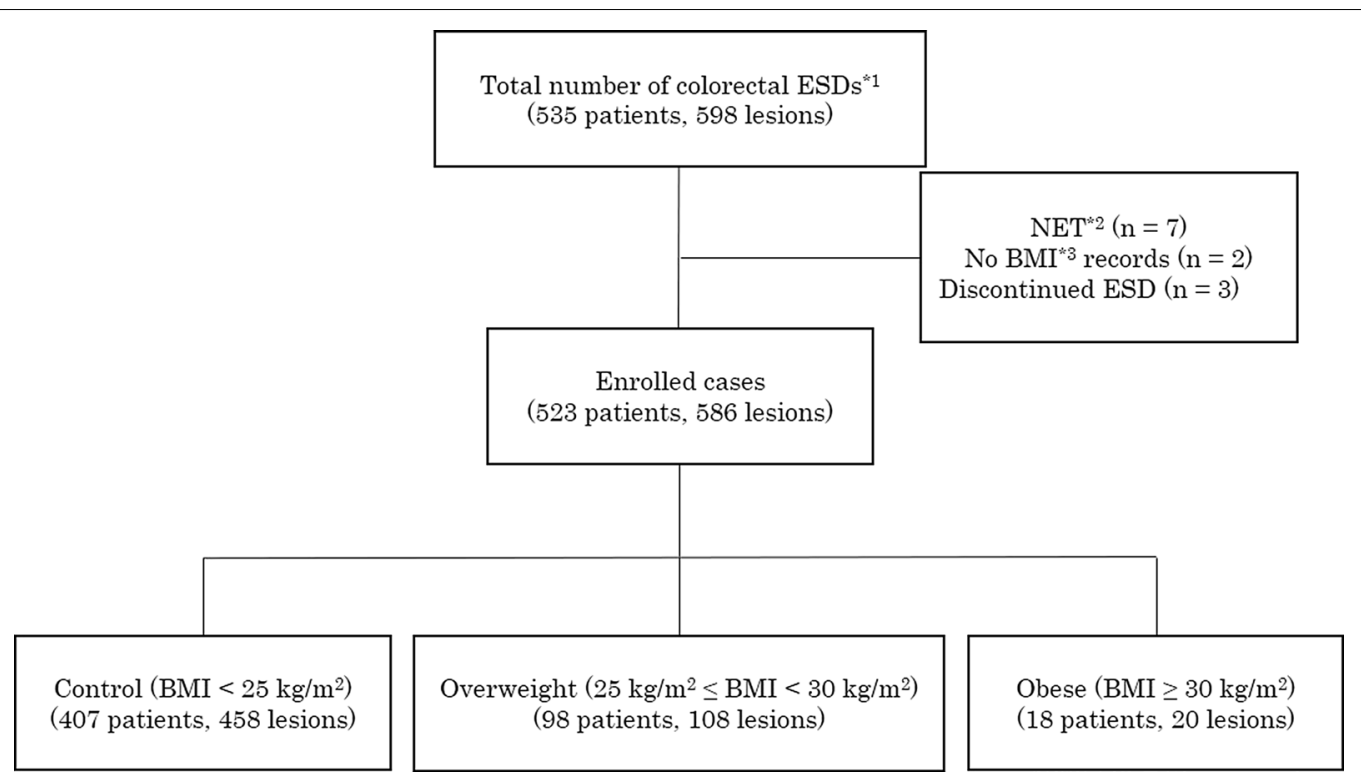

Fig. 2 Patients who had underwent colorectal ESD and did not meet the exclusion criteria were divided into three groups according to their BMI. *1 Endoscopic submucosal dissection. *2 Neuroendocrine tumor. *3 Body mass index

difference in the cecal insertion time during the preoperative colonoscopy was seen. Other patient characteristics did not differ among the groups. The lesion characteristics, locations, macroscopic types, and pathologies were similar among the groups.

\section{Clinical outcomes}

Table 2 presents the clinical outcomes of colorectal ESD according to the body mass index groups. En bloc resection was achieved in 585 (99.8\%) patients, complete resection was achieved in 579 (98.8\%) patients, and curable resection was achieved in $573(97.8 \%)$ patients. No significant differences in the en bloc and curable resection rates were seen among the groups. The median specimen size was $32.0(24.0-40.0) \mathrm{mm}$, and the median lesion size was $24.0(20.0-30.0) \mathrm{mm}$. No significant differences in these parameters were seen among the groups. In the pathological results for the resected specimens, the frequencies of vertical margin positivity, horizontal margin positivity, lymphovascular invasion, tumor budding, and the submucosal invasion of tumor cells did not differ significantly among the groups. The median procedure time was $31.0(20.0-53.0) \mathrm{min}$, and $62(10.4 \%)$ cases required a procedure time of longer than $90 \mathrm{~min}$. No significant difference in procedure time was seen among the groups. Regarding monitoring during the procedure, no significant difference in a systolic blood pressure variability of more than $50 \mathrm{mmHg}$ or the incidence of bradycardia was seen. The number of cases with hypoxemia differed significantly among the groups, with a higher BMI associated with a greater likelihood of hypoxemia (123 [30.2\%] vs. 43 [43.9\%] vs. 10 [55.6\%], $P=0.005)$. Nevertheless, the amount of sedative per body weight was significantly higher in the group with a lower BMI (flunitrazepam: $1.75 \times 10^{-2}\left[1.27 \times 10^{-2}-2.34 \times 10^{-2}\right] \mathrm{mg} /$ $\mathrm{kg}$ vs. $1.48 \times 10^{-2}\left[1.08 \times 10^{-2}-2.03 \times 10^{-2}\right] \mathrm{mg} / \mathrm{kg}$ vs. $1.16 \times 10^{-2}\left[0.98 \times 10^{-2}-1.54 \times 10^{-2}\right] \mathrm{mg} / \mathrm{kg}, \quad P<0.001$; pethidine: $0.63[0.55-0.72] \mathrm{mg} / \mathrm{kg}$ vs. 0.50 [0.46-0.56] $\mathrm{mg} / \mathrm{kg}$ vs. 0.39 [0.32-0.45] mg/kg, $P<0.001)$. Perforation was observed in four cases and bleeding in seven cases, but no significant difference was seen among the groups. All the patients with delayed bleeding were treated with endoscopic hemostasis. Three of the patients with perforation were treated with endoscopic closure, and one underwent emergency surgery for delayed perforation. After ESD, a few delayed events occurred. One patient developed a pulmonary embolism and one developed sick sinus syndrome in the overweight group, while one patient developed a pulmonary embolism and one developed subarachnoid hemorrhage in the control group. During the follow-up period (range, 2-87 months), none of the patients died as a result of colorectal cancer, although 10 patients died as a result of other diseases (5 from lung cancer, 1 from malignant lymphoma, and 4 from unknown causes).

Table 3 presents the results of the present study limited to cases treated by trainees with less than 100 cases of colorectal ESD experience. The number of cases that required a procedure time of longer than $90 \mathrm{~min}$ was significantly larger in the group with a higher BMI 
Table 1 Baseline characteristics of the patients and lesions

\begin{tabular}{|c|c|c|c|c|c|}
\hline & Total & $\begin{array}{l}\text { Control } \\
\left(\mathrm{BMI}<25 \mathrm{~kg} / \mathrm{m}^{2}\right)\end{array}$ & $\begin{array}{l}\text { Overweight } \\
(25 \mathrm{~kg} / \\
\mathrm{m}^{2} \leq \mathrm{BMI}<30 \mathrm{~kg} / \\
\left.\mathrm{m}^{2}\right)\end{array}$ & $\begin{array}{l}\text { Obese } \\
\left(\mathrm{BMI} \geq 30 \mathrm{~kg} / \mathrm{m}^{2}\right)\end{array}$ & $P$ value \\
\hline Patients $(n)$ & 523 & 407 & 98 & 18 & \\
\hline Age (median $\left[\mathrm{QQR}^{* 1}\right]$ ) & $70.0(62.5-77.0)$ & $71.0(63.0-77.0)$ & $69.5(61.5-75.0)$ & $67.5(59.3-80.0)$ & 0.354 \\
\hline Sex, male $(n)$ & $300(57.4 \%)$ & $227(55.8 \%)$ & $64(65.3 \%)$ & $9(50.0 \%)$ & 0.188 \\
\hline $\mathrm{BMI}^{* 2}\left(\mathrm{~kg} / \mathrm{m}^{2}\right.$, median [IQR]) & $22.8(20.3-24.8)$ & $21.7(19.8-23.4)$ & $26.3(25.6-27.3)$ & $32.4(31.2-34.0)$ & $<0.001$ \\
\hline Cecal insertion time ${ }^{* 3}$ (min, median [IQR]) & $5.5(4.0-7.0)$ & $6.0(4.0-7.5)$ & $5.0(3.0-6.0)$ & $5.0(4.0-5.25)$ & 0.086 \\
\hline \multicolumn{6}{|l|}{ Comorbidities $(n)$} \\
\hline Hypertension & $236(45.1 \%)$ & $174(42.8 \%)$ & $53(54.1 \%)$ & $9(50.0 \%)$ & 0.118 \\
\hline Diabetes & $66(12.6 \%)$ & $46(11.3 \%)$ & $18(18.4 \%)$ & $2(11.1 \%)$ & 0.164 \\
\hline Cardiovascular disease & $17(3.3 \%)$ & $14(3.4 \%)$ & $2(2.0 \%)$ & $1(5.6 \%)$ & 0.668 \\
\hline Cerebrovascular disease & $53(10.1 \%)$ & $42(11.5 \%)$ & $7(7.1 \%)$ & $4(22.2 \%)$ & 0.145 \\
\hline Liver cirrhosis & $9(1.7 \%)$ & $7(1.7 \%)$ & $2(2.0 \%)$ & $0(0.0 \%)$ & 0.829 \\
\hline Chronic renal failure & $15(2.9 \%)$ & $14(3.4 \%)$ & $1(1.0 \%)$ & $0(0.0 \%)$ & 0.331 \\
\hline Respiratory disease ${ }^{* 4}$ & $21(4.0 \%)$ & $19(4.7 \%)$ & $2(2.0 \%)$ & $0(0.0 \%)$ & 0.334 \\
\hline Antithrombotic medication ( $n$ ) & $90(17.2 \%)$ & $71(17.4 \%)$ & $15(15.3 \%)$ & $4(22.2 \%)$ & 0.747 \\
\hline Lesion $(n)$ & 586 & 458 & 108 & 20 & \\
\hline Location (n) & & & & & 0.809 \\
\hline Cecum & $84(14.3 \%)$ & $66(14.4 \%)$ & $13(12.0 \%)$ & $5(25.0 \%)$ & \\
\hline Ascending & $141(24.1 \%)$ & $108(23.6 \%)$ & $28(25.9 \%)$ & $5(25.0 \%)$ & \\
\hline Transverse & $131(22.4 \%)$ & $100(21.8 \%)$ & $26(24.1 \%)$ & $5(25.0 \%)$ & \\
\hline Descending & $47(8.0 \%)$ & $35(7.6 \%)$ & $11(10.2 \%)$ & $1(5.0 \%)$ & \\
\hline Sigmoid & $84(14.3 \%)$ & $66(14.4 \%)$ & 15 (13.9\%) & $3(15.0 \%)$ & \\
\hline Rectum & 99 (16.9\%) & $83(18.1 \%)$ & 15 (13.9\%) & $1(5.0 \%)$ & \\
\hline Macroscopic type $(n)$ & & & & & 0.795 \\
\hline Protruded & 99 (16.9\%) & $78(17.0 \%)$ & $17(15.7 \%)$ & $4(20.0 \%)$ & \\
\hline Elevated & $482(82.2 \%)$ & 375 (81.9\%) & $91(84.3 \%)$ & $16(80.0 \%)$ & \\
\hline Depressed & $5(0.9 \%)$ & $5(1.1 \%)$ & $0(0.0 \%)$ & $0(0.0 \%)$ & \\
\hline Pathologycal diagnosis (n) & & & & & 0.636 \\
\hline Adenoma & $367(62.6 \%)$ & $293(64.0 \%)$ & $64(59.3 \%)$ & $10(50.0 \%)$ & \\
\hline Tis & $165(28.2 \%)$ & $123(26.9 \%)$ & $34(31.5 \%)$ & $8(40.0 \%)$ & \\
\hline $\mathrm{T} 1$ & $54(9.2 \%)$ & $42(9.2 \%)$ & $10(9.3 \%)$ & $2(10.0 \%)$ & \\
\hline
\end{tabular}

* 1 Interquartile range

* 2 Body mass index

* 3 Cecal insertion time of the colonoscopy for pre-ESD observation

* 4 interstitial lung disease or chronic obstructive pulmonary disease

(27 [10.8\%] vs. 14 [21.9\%] vs. 3 [25.0\%], $P=0.033$ ). No significant differences in the self-completion rates, en bloc resection rates, mean specimen sizes, mean lesion sizes, and pathological results were seen. The incidence of hypoxemia was not significantly different, but a higher BMI tended to increase the risk of hypoxemia (77 [34.8\%] vs. 27 [48.2\%] vs. 6 [54.5\%], $P=0.096$ ). Similar to the overall analysis, the amount of pethidine per body weight was significantly lower in the group with a higher BMI $(0.61[0.54-0.69] \mathrm{mg} / \mathrm{kg}$ vs. $0.49[0.47-$ $0.56] \mathrm{mg} / \mathrm{kg}$ vs. $0.38[0.33-0.46] \mathrm{mg} / \mathrm{kg}, P<0.001)$.
No significant differences in complications or delayed events were seen.

\section{Discussion}

In this study, we analyzed the impact of obesity on the performance of colorectal ESD. No significant differences in short-term clinical outcomes, such as the en bloc resection rate, pathological results and adverse events, were seen between obese patients and non-obese patients. Although the impact of obesity on the performance of colorectal ESD has not been previously reported, the 
Table 2 Clinical outcomes of colorectal ESD according to body mass index groups

\begin{tabular}{|c|c|c|c|c|c|}
\hline & Total & $\begin{array}{l}\text { Control } \\
\left(B M I^{* 8}<25 \mathrm{~kg} / \mathrm{m}^{2}\right)\end{array}$ & $\begin{array}{l}\text { Overweight } \\
\left(25 \mathrm{~kg} / \mathrm{m}^{2} \leq \mathrm{BMI}<30 \mathrm{~kg} / \mathrm{m}^{2}\right)\end{array}$ & $\begin{array}{l}\text { Obese } \\
\left(B M I \geq 30 \mathrm{~kg} / \mathrm{m}^{2}\right)\end{array}$ & $P$ value \\
\hline Lesion $(n)$ & 586 & 458 & 108 & 20 & \\
\hline Operator, trainee ( $n$ ) & $327(55.8 \%)$ & $251(54.8 \%)$ & $64(59.3 \%)$ & $12(60.0 \%)$ & 0.653 \\
\hline $\begin{array}{l}\text { Procedure time (min, median } \\
\left.\left[\mathrm{IQR}^{*}\right]\right] \text { ) }\end{array}$ & $31.0(20.0-53.0)$ & $30.0(20.0-50.8)$ & $33.5(22.0-60.0)$ & $35.5(24.5-48.0)$ & 0.395 \\
\hline $\begin{array}{l}\text { Longer procedure time } \\
\qquad(\geq 90 \mathrm{~min})(n)\end{array}$ & $62(10.6 \%)$ & $42(9.2 \%)$ & $17(15.7 \%)$ & $3(15.0 \%)$ & 0.110 \\
\hline $\begin{array}{l}\text { Specimen size (mm, median } \\
{[\text { [QR] })}\end{array}$ & $32.0(24.0-40.0)$ & $32.0(25.0-40.0)$ & $32.0(26.0-40.0)$ & $33.0(24.0-41.3)$ & 0.673 \\
\hline $\begin{array}{l}\text { Lesion size (mm, median } \\
\text { [IQR]) }\end{array}$ & $24.0(20.0-30.0)$ & $23.0(20.0-30.0)$ & $25.0(20.0-32.0)$ & $23.0(16.0-32.5)$ & 0.503 \\
\hline Use of hemostatic forceps $(n)$ & $53(9.0 \%)$ & $41(9.4 \%)$ & $9(8.3 \%)$ & $3(15.0 \%)$ & 0.627 \\
\hline Fibrosis $(n)$ & & & & & 0.878 \\
\hline Fo & $472(80.5 \%)$ & $368(80.3 \%)$ & 89 (82.4\%) & $15(75.0 \%)$ & \\
\hline F1 & $76(13.0 \%)$ & $59(12.9 \%)$ & $14(13.0 \%)$ & $3(15.0 \%)$ & \\
\hline $\mathrm{F} 2$ & $38(6.5 \%)$ & $31(6.8 \%)$ & $5(4.6 \%)$ & $2(10.0 \%)$ & \\
\hline En bloc resection ( $n$ ) & $585(99.8 \%)$ & $458(100 \%)$ & 107 (99.1\%) & $20(100.0 \%)$ & 0.109 \\
\hline Complete resection ( $n$ ) & $579(98.8 \%)$ & 454 (99.1\%) & $105(97.2 \%)$ & $20(100.0 \%)$ & 0.230 \\
\hline Curative resection (n) & $573(97.8 \%)$ & 449 (98.0\%) & $104(96.3 \%)$ & $20(100.0 \%)$ & 0.430 \\
\hline Vertical margin positivity $(n)$ & $6(1.0 \%)$ & $4(0.9 \%)$ & $2(1.9 \%)$ & $0(0.0 \%)$ & 0.595 \\
\hline $\begin{array}{l}\text { Horizontal margin positiv- } \\
\text { ity }(n)\end{array}$ & $1(0.2 \%)$ & $0(0.0 \%)$ & $1(0.9 \%)$ & $0(0.0 \%)$ & 0.109 \\
\hline Tumor budding $(n)$ & & & & & 0.617 \\
\hline Grade 1 & $52(8.9 \%)$ & $40(8.7 \%)$ & $10(9.3 \%)$ & $2(10.0 \%)$ & \\
\hline Grade 2, 3 & $2(0.3 \%)$ & $2(0.4 \%)$ & $0(0.0 \%)$ & $0(0.0 \%)$ & \\
\hline Patients $(n)$ & 523 & 407 & 98 & 18 & \\
\hline \multicolumn{6}{|c|}{ Change of vital signs during ESD ( $n$ ) } \\
\hline SBP fluctuation ${ }^{* 2}$ & $110(21.0 \%)$ & $88(21.6 \%)$ & $27(27.6 \%)$ & $5(27.8 \%)$ & 0.403 \\
\hline Bradycardia $^{* 3}$ & $30(5.7 \%)$ & $24(5.9 \%)$ & $5(5.1 \%)$ & $1(5.6 \%)$ & 0.905 \\
\hline Hypoxemia $^{* 4}$ & $176(33.7 \%)$ & $123(30.2 \%)$ & $43(43.9 \%)$ & $10(55.6 \%)$ & 0.005 \\
\hline $\begin{array}{l}\text { Amount of flunitrazepam } \\
\text { (mg/kg, median [IQR]) }\end{array}$ & $\begin{array}{l}1.67 \times 10^{-2} \\
\left(1.22 \times 10^{-2}-2.27 \times 10^{-2}\right)\end{array}$ & $\begin{array}{l}1.75 \times 10^{-2} \\
\left(1.27 \times 10^{-2}-2.34 \times 10^{-2}\right)\end{array}$ & $\begin{array}{l}1.48 \times 10^{-2} \\
\left(1.08 \times 10^{-2}-2.03 \times 10^{-2}\right)\end{array}$ & $\begin{array}{l}1.16 \times 10^{-2} \\
\left(0.98 \times 10^{-2}-1.54 \times 10^{-2}\right)\end{array}$ & $<0.001$ \\
\hline $\begin{array}{l}\text { Amount of pethidine } \\
(\mathrm{mg} / \mathrm{kg} \text {, median [IQR]) }\end{array}$ & $0.59(0.51-0.69)$ & $0.63(0.55-0.72)$ & $0.50(0.46-0.56)$ & $0.39(0.32-0.45)$ & $<0.001$ \\
\hline Delayed bleeding (n) & $7(1.3 \%)$ & $7(1.7 \%)$ & $0(0.0 \%)$ & $0(0.0 \%)$ & 0.314 \\
\hline Perforation ( $n$ ) & $4(0.8 \%)$ & $3(0.7 \%)$ & $1(1.0 \%)$ & $0(0.0 \%)$ & 0.893 \\
\hline $\operatorname{PECS}^{* 5}(n)$ & $5(1.0 \%)$ & $3(2.6 \%)$ & $2(2.0 \%)$ & $0(0.0 \%)$ & 0.450 \\
\hline \multicolumn{6}{|l|}{ Delayed event ${ }^{*} 6(n)$} \\
\hline Pulmonary embolism & $2(0.4 \%)$ & $1(0.2 \%)$ & $1(1.0 \%)$ & $0(0.0 \%)$ & 0.518 \\
\hline Sick sinus syndrome & $1(0.2 \%)$ & $0(0.0 \%)$ & $1(1.0 \%)$ & $0(0.0 \%)$ & 0.114 \\
\hline Subarachnoid hemorrhage & $1(0.2 \%)$ & $1(0.2 \%)$ & $0(0.0 \%)$ & $0(0.0 \%)$ & 0.867 \\
\hline $\begin{array}{l}\text { Hospitalization }{ }^{* 7} \\
\text { (days, median [IQR]) }\end{array}$ & $5.0(5.0-5.0)$ & $5.0(5.0-5.0)$ & $5.0(5.0-5.0)$ & $5.0(5.0-5.0)$ & 0.927 \\
\hline
\end{tabular}

\footnotetext{
* 1 Interquartile range

* 2 Systolic blood pressure fluctuation of more than $50 \mathrm{~mm} \mathrm{Hg}$

* 3 Heart rate decreasing below $50 / \mathrm{min}$

* 4 Oxygen desaturation below $90 \%$

* 5 Post-ESD coagulation syndrome

* 6 Adverse events other than bleeding and perforation that occurred within 30 days after ESD

* 7 Number of hospitalization days

* 8 Body mass index
} 
Table 3 Clinical outcome of colorectal ESD performed by trainees according to body mass index groups

\begin{tabular}{|c|c|c|c|c|c|}
\hline & Total & $\begin{array}{l}\text { Control } \\
\left(B M I^{* 8}<25 \mathrm{~kg} / \mathrm{m}^{2}\right)\end{array}$ & $\begin{array}{l}\text { Overweight } \\
\left(25 \mathrm{~kg} / \mathrm{m}^{2} \leq \mathrm{BMI}<30 \mathrm{~kg} /\right. \\
\left.\mathrm{m}^{2}\right)\end{array}$ & $\begin{array}{l}\text { Obese } \\
\left(B M I \geq 30 \mathrm{~kg} / \mathrm{m}^{2}\right)\end{array}$ & $P$ value \\
\hline Lesion ( $n)$ & 327 & 251 & 64 & 12 & \\
\hline $\begin{array}{l}\text { Procedure time (min, } \\
\text { median }\left[\mathrm{IQR}^{*}\right] \text { ) }\end{array}$ & $40.0(26.0-60.0)$ & $40.0(25.0-59.0)$ & $47.0(30.0-74.3)$ & $38.0(30.8-63.0)$ & 0.150 \\
\hline $\begin{array}{l}\text { Longer procedure time } \\
\qquad(\geq 90 \mathrm{~min})(n)\end{array}$ & $44(13.5 \%)$ & $27(10.8 \%)$ & $14(21.9 \%)$ & $3(25.0 \%)$ & 0.033 \\
\hline $\begin{array}{l}\text { Specimen size (mm, min, } \\
\text { median [IQR]) }\end{array}$ & $30.0(24.5-38.0)$ & $30.0(24.0-37.0)$ & $30.0(25.8-40.0)$ & $29.5(23.5-36.0)$ & 0.456 \\
\hline Lesion size $(\mathrm{mm}$ mean $\pm \mathrm{SD})$ & $22.0(19.0-29.0)$ & $22.0(18.5-28.0)$ & $24.5(19.8-32.0)$ & $20.0(16.0-29.0)$ & 0.274 \\
\hline Fibrosis (n) & & & & & 0.942 \\
\hline F0 & $277(84.7 \%)$ & $214(85.3 \%)$ & $53(82.8 \%)$ & $10(83.3 \%)$ & \\
\hline F1 & $33(10.1 \%)$ & $24(9.6 \%)$ & $8(12.5 \%)$ & $1(8.3 \%)$ & \\
\hline F2 & $17(5.2 \%)$ & $13(5.2 \%)$ & $3(4.7 \%)$ & $1(8.3 \%)$ & \\
\hline Self-completion ( $n$ ) & $279(85.3 \%)$ & $218(86.0 \%)$ & $51(79.7 \%)$ & $10(83.3 \%)$ & 0.345 \\
\hline En bloc resection (n) & $326(99.7 \%)$ & $251(100 \%)$ & $63(98.4 \%)$ & $12(100.0 \%)$ & 0.127 \\
\hline Complete resection ( $n$ ) & $322(98.5 \%)$ & $249(99.2 \%)$ & $61(95.3 \%)$ & $12(100.0 \%)$ & 0.070 \\
\hline Curative resection (n) & $317(96.9 \%)$ & $245(97.6 \%)$ & $60(93.8 \%)$ & $12(100.0 \%)$ & 0.228 \\
\hline Vertical margin positivity $(n)$ & $4(1.2 \%)$ & $2(0.8 \%)$ & $2(3.1 \%)$ & $0(0.0 \%)$ & 0.295 \\
\hline $\begin{array}{l}\text { Horizontal margin positivity } \\
(n)\end{array}$ & $1(0.3 \%)$ & $0(0.0 \%)$ & $1(1.6 \%)$ & $0(0.0 \%)$ & 0.127 \\
\hline Tumor budding $(n)$ & & & & & 0.862 \\
\hline Grade 1 & $29(8.9 \%)$ & $21(8.4 \%)$ & $6(9.4 \%)$ & $2(16.7 \%)$ & \\
\hline Grade 2, 3 & $1(0.3 \%)$ & $1(0.4 \%)$ & $0(0.0 \%)$ & $0(0.0 \%)$ & \\
\hline Patients $(n)$ & 288 & 221 & 56 & 11 & \\
\hline \multicolumn{6}{|c|}{ Change of vital signs during ESD (n) } \\
\hline SBP fluctuation ${ }^{* 2}$ & $77(26.7 \%)$ & $57(25.8 \%)$ & $17(30.4 \%)$ & $3(27.3 \%)$ & 0.788 \\
\hline Bradycardia ${ }^{* 3}$ & $21(7.3 \%)$ & $16(7.2 \%)$ & $5(8.9 \%)$ & $0(0.0 \%)$ & 0.580 \\
\hline Hypoxemia ${ }^{*}{ }_{4}$ & $110(38.2 \%)$ & $77(34.8 \%)$ & $27(48.2 \%)$ & $6(54.5 \%)$ & 0.096 \\
\hline $\begin{array}{l}\text { Amount of flunitrazepam } \\
\left(\mathrm{mg} / \mathrm{kg} \text {, median }[\mathrm{IQR}]^{* 4}\right)\end{array}$ & $\begin{array}{l}1.73 \times 10^{-2} \\
\left(1.27 \times 10^{-2}-2.26 \times 10^{-2}\right)\end{array}$ & $\begin{array}{l}1.77 \times 10^{-2} \\
\left(1.32 \times 10^{-2}-2.29 \times 10^{-2}\right)\end{array}$ & $\begin{array}{l}1.55 \times 10^{-2} \\
\left(1.17 \times 10^{-2}-2.23 \times 10^{-2}\right)\end{array}$ & $\begin{array}{l}1.33 \times 10^{-2} \\
\left(1.21 \times 10^{-2}-1.98 \times 10^{-2}\right)\end{array}$ & 0.197 \\
\hline $\begin{array}{l}\text { Amount of pethidine } \\
\text { (mg/kg, median [IQR]) }\end{array}$ & $0.58(0.51-0.66)$ & $0.61(0.54-0.69)$ & $0.49(0.47-0.56)$ & $0.38(0.33-0.46)$ & $<0.001$ \\
\hline Delayed bleeding $(n)$ & $6(2.1 \%)$ & $6(2.7 \%)$ & $0(0.0 \%)$ & $0(0.0 \%)$ & 0.395 \\
\hline Perforation $(n)$ & $3(1.0 \%)$ & $2(0.9 \%)$ & $1(1.8 \%)$ & $0(0.0 \%)$ & 0.796 \\
\hline $\operatorname{PECS}^{* 5}(n)$ & $3(1.0 \%)$ & $1(0.5 \%)$ & $2(3.6 \%)$ & $0(0.0 \%)$ & 0.114 \\
\hline \multicolumn{6}{|l|}{ Delayed event ${ }^{* 6}(n)$} \\
\hline Pulmonary embolism & $1(0.3 \%)$ & $0(0.0 \%)$ & $1(1.8 \%)$ & $0(0.0 \%)$ & 0.125 \\
\hline Sick sinus syndrome & $1(0.3 \%)$ & $0(0.0 \%)$ & $1(1.8 \%)$ & $0(0.0 \%)$ & 0.125 \\
\hline $\begin{array}{l}\text { Hospitalization }{ }^{* 7} \text { (days, } \\
\text { median [IQR]) }\end{array}$ & $5.0(5.0-5.0)$ & $5.0(5.0-5.0)$ & $5.0(5.0-5.0)$ & $5.0(5.0-5.0)$ & 0.721 \\
\hline
\end{tabular}

* 1 Interquartile range

* 2 Systolic blood pressure fluctuation of more than $50 \mathrm{~mm} \mathrm{Hg}$

* 3 Heart rate decreasing below $50 / \mathrm{min}$

${ }^{*} 4$ Oxygen desaturation below $90 \%$

* 5 Post-ESD coagulation syndrome

* 6 Adverse events other than bleeding and perforation that occurred within 30 days after ESD

* 7 Number of hospitalization days

* 8 Body mass index 
present study showed that ESD is safe and effective for obese patients. However, obesity was significantly associated with changes in the respiratory status during ESD.

A previous study showed that risk factors for hypoxemia associated with sedation during endoscopy were an older age, the presence of major comorbidities, the total pethidine dose, and obesity [21-24]. Although these factors have been reviewed for routine endoscopy, few reports have examined the risk of hypoxemia during colorectal ESD. In the present study, obese patients were shown to have a significantly increased incidence of hypoxemia during colorectal ESD. The presence of obstructive sleep apnea (OSA) is reportedly related to hypoxemia during the sedation of morbidly obese patients [25]. Although we did not examine the presence of OSA in this study, obesity is generally a risk factor for sleep apnea syndrome, which may have affected the present results.

In our hospital, if hypoxemia occurs during colorectal ESD, oxygen is first administered through a nasal cannula. In the cases with hypoxemia reported here, the oxygen saturation improved in all the cases with the administration of oxygen, and the administration of a sedative antagonist during the procedure or an emergency tracheal intubation was not required in any of the cases. In our hospital, anesthesiologists usually do not attend colorectal ESD procedures, and the sedation is performed by the endoscopists. However, the patient's vital signs are constantly monitored by doctors and nurses other than the doctor performing the procedure, and sedatives are added as needed based on the patient's condition. Since the amount of sedative was intentionally reduced in obese patients because of their susceptibility to hypoxemia, the amount of sedatives per kilogram of body weight thought to be significantly lower in the group with a higher BMI in this study.

On the other hand, when the procedures were performed by trainees, the number of cases that required a long procedure time was significantly larger in the group with a higher BMI. This result may reflect the difficulty of performing colorectal ESD in obese patients, particularly for trainees. In previous reports, predictors of colorectal ESD difficulty included a location in the right colon, tumor size, submucosal infiltration, fibrosis, nonexperienced physician, and manipulation difficulties, but no previous report has shown a relationship with patient obesity $[8,15,26,27]$. In these reports, scope manipulation difficulties were defined as either paradoxical movements, poor control because of adhesions, or the motion of the lesion as a result of heartbeats or breathing. In obese patients, previous studies have suggested that scope insertion can be difficult because of the formation of loops in the scope, difficulties in changing the patient's position, ineffective abdominal pressure, and inadequate bowel preparation [28-30]. In addition, obese patients may experience sudden respiratory changes associated with OSA. The above factors are thought to be responsible for the poor scope maneuverability during ESD in obese patients. However, regarding the difficulty of insertion, in this study, no significant difference was seen among the groups in terms of the preoperative insertion time. In previous studies examining colonoscope insertion, not only obesity, but also being underweight was reported to be a factor in insertion difficulty [28-30]; therefore, the results for insertion time in the present study might also have been affected by patients who were underweight. Kang et al. reported that the procedure time was significantly longer in obese patients undergoing gastric ESD [10]. According to their study, a large amount of submucosal fat tissue in obese patients was thought to make dissection more difficult because of poor vision arising from burnt fat or aerosolized fat droplets; a similar situation might have occurred in the present study.

The procedure time for trainees operating on obese patients was thought to be prolonged in this study for the above reasons. Because the usefulness of overtube during ESD in the right-side colon, where scopes tend to form loops, has also been reported [31], the use of an overtube may be one option for performing ESD in the right-side colon of obese patients.

In our hospital, when a trainee performs ESD, an expert always supervises the procedure. As a result, no significant difference in the en bloc resection rate or the number of complications were seen in the present study. However, a long procedure time has been reported to be a risk factor for perforation [32]. In facilities where experts routinely observe procedures, the replacement of the physicians by the expert observing the procedure might be advisable if difficulties are anticipated so as to minimize the procedure time and reduce the risk of perforation.

Regarding delayed events, one patient developed sick sinus syndrome, two patients developed pulmonary embolisms, and one patient developed a subarachnoid hemorrhage. Although no significant differences were seen among the groups, some reports have described significantly higher risks of cardiovascular complications in obese surgical patients [1-6]. Therefore, further investigation is needed to determine the rate of cardiovascular complications in obese patients undergoing colorectal ESD.

The present study had some limitations. This was a retrospective, single-institution study, and most of the patients analyzed in this study were Japanese. Therefore, these results may not be applicable to other ethnicities. 
A multicenter, prospective study with a larger number of cases is needed to validate our results. Second, this study might not have assessed the effects of visceral fat accurately because BMI is not always correlated with the amount of fat in the body. In addition, since scope maneuverability and the degree of fatty tissue in the submucosa during ESD could not be studied retrospectively in this study, the impact of poor scope maneuverability and an abundance of fatty tissue on the treatment of obese patients remains unclear. In order to clarify the effects of these factors, further examination about the evaluation method of the degree of fatty tissue and the scope maneuverability are needed.

\section{Conclusions}

In conclusion, colorectal ESD was safe and effective in obese patients. However, ESD in patients with obesity requires attention to changes in respiratory conditions and potential difficulties that might be encountered by trainees performing such procedure.

\section{Abbreviations}

ESD: Endoscopic submucosal dissection; BMI: Body mass index; $\mathrm{SpO}_{2}$ : Percutaneous arterial oxygen saturation; OSA: Obstructive sleep apnea.

\section{Acknowledgements}

The authors would like to thank the staff of the participating institutions for their support in recruiting eligible patients, and as well as the patients who participated in this study.

\section{Authors' contributions}

JT, HC, TH, TG and AN conceived of the study. JT, HC, NO, JA, KA, HK and MN performed the ESD. JT, NO, JA, KA, HK and MN recruited the study participants. Analysis and interpretation of the data was conducted by JT and HC. All the authors have read the final manuscript and have approved its submission for publication. All authors read and approved the final manuscript.

\section{Funding}

This study received no external funding. There was no sponsor for this study.

\section{Availability of data and materials}

The datasets used and/or analyzed during the current study are available from the corresponding author upon reasonable request.

\section{Ethics approval and consent to participate}

The study was conducted in accordance with the principles laid down in the Declaration of Helsinki, and with the approval of the Institutional Review Board of Omori Red Cross Hospital (No. 19-31). Informed consent was obtained from all individual participants included in the study.

\section{Consent for publication}

Patients signed informed consent forms regarding the publication of their data and photographs.

\section{Competing of interests}

The authors have no conflicts of interest to declare.

\section{Author details}

${ }^{1}$ Department of Gastroenterology, Omori Red Cross Hospital, 4-30-1, Chuo, Ota-Ku, Tokyo 143-0024, Japan. ${ }^{2}$ Department of Gastroenterology and Hepatology, Yokohama City University Graduate School of Medicine, 3-9, Fukuura, Kanazawa-Ku, Yokohama 236-0004, Japan.
Received: 17 October 2020 Accepted: 2 February 2021

Published online: 16 February 2021

\section{References}

1. Yasunaga H, Horiguchi H, Matsuda S, Fushimi K, Hashimoto H, Ayanian JZ. Body mass index and outcomes following gastrointestinal cancer surgery in Japan. Br J Surg. 2013;100:1335-43.

2. Zhai TS, Kang Y, Ren WH, Liu Q, Liu C, Mao WZ. Elevated visceral fat area is associated with adverse postoperative outcome of radical colectomy for colon adenocarcinoma patients. ANZ J Surg. 2019;89:E368-72.

3. Ozoya OO, Siegel EM, Srikumar T, Bloomer AM, DeRenzis A, Shibata D. Quantitative assessment of visceral obesity and postoperative colon cancer outcomes. J Gastrointest Surg. 2017;21:534-42.

4. Amri R, Bordeianou LG, Sylla P, Berger DL. Obesity, outcomes and quality of care: body mass index increases the risk of wound-related complications in colon cancer surgery. Am J Surg. 2014;207:17-23.

5. Cakir H, Heus $\mathrm{C}$, van der Ploeg TJ, Houdijk AP. Visceral obesity determined by $\mathrm{CT}$ scan and outcomes after colorectal surgery; a systematic review and meta-analysis. Int J Colorectal Dis. 2015;30:875-82.

6. Poelemeijer YQM, Lijftogt N, Detering R, Fiocco M, Tollenaar RAEM, Wouters MWJM. Obesity as a determinant of perioperative and postoperative outcome in patients following colorectal cancer surgery: a populationbased study (2009-2016). Eur J Surg Oncol. 2018;44:1849-57.

7. Ohata K, Nonaka K, Minato Y, Misumi Y, Tashima T, Shozushima M, et al. Endoscopic submucosal dissection for large colorectal tumor in a Japanese general hospital. J Oncol. 2013. https://doi.org/10.1155/2013/21867 0.

8. Hayashi N, Tanaka S, Nishiyama S, Terasaki M, Nakadoi K, Oka S, et al. Predictors of incomplete resection and perforation associated with endoscopic submucosal dissection for colorectal tumors. Gastrointest Endosc. 2014;79:427-35.

9. Chiba H, Tachikawa J, Arimoto J, Ashikari K, Kuwabara H, Nakaoka M, et al. Predictive factors of mild and severe fibrosis in colorectal endoscopic submucosal dissection. Dig Dis Sci. 2020;65:232-42.

10. Kang D, Ha SE, Park JM, Yoon SB, Lee HH, Lim CH, et al. Body mass index and clinical outcomes from endoscopic submucosal dissection of gastric neoplasia. Dig Dis Sci. 2017;62:1657-65.

11. Tanaka H, Oka S, Tanaka S, Matsumoto K, Boda K, Yamashita K, et al. Dual red imaging maintains clear visibility during colorectal endoscopic submucosal dissection. Dig Dis Sci. 2019;64:224-31.

12. Yoshida N, Naito Y, Hirose R, Ogiso K, Siah KT, Inada Y, et al. Risk of lens cloudiness during colorectal endoscopic submucosal dissection and ability of a novel lens cleaner to maintain and restore endoscopic view. Dig Endosc. 2015;27:609-17.

13. WHO. Obesity: preventing and managing the global epidemic. Geneva: World Health Organization Technical Report Series No. 894; 2000.

14. Yoshida M, Takizawa K, Nonaka S, Shichijo S, Suzuki S, Sato C, et al. Conventional versus traction-assisted endoscopic submucosal dissection for large esophageal cancers: a multicenter, randomized controlled trial (with video). Gastrointest Endosc. 2020;91(55-65):e2.

15. Chiba H, Tachikawa J, Kurihara D, Ashikari K, Goto T, Takahashi A, et al. Safety and efficacy of simultaneous colorectal ESD for large synchronous colorectal lesions. Endosc Int Open. 2017;5:E595-602.

16. Arimoto J, Higurashi T, Kato S, Fuyuki A, Ohkubo H, Nonaka T, et al. Risk factors for post-colorectal endoscopic submucosal dissection (ESD) coagulation syndrome: a multicenter, prospective, observational study. Endosc Int Open. 2018;6:E342-9.

17. Matsumoto A, Tanaka S, Oba S, Kanao H, Oka S, Yoshihara M, et al. Outcome of endoscopic submucosal dissection for colorectal tumors accompanied by fibrosis. Scand J Gastroenterol. 2010;45:1329-37.

18. Chiba H, Ohata K, Tachikawa J, Arimoto J, Ashikari K, Kuwabara H, et al. Delayed bleeding after colorectal endoscopic submucosal dissection: when is emergency colonoscopy needed? Dig Dis Sci. 2019;64:880-7.

19. Tanaka S, Kashida H, Saito Y, Yahagi N, Yamano H, Saito S, et al. JGES guidelines for colorectal endoscopic submucosal dissection/endoscopic mucosal resection. Dig Endosc. 2015;27:417-34.

20. Hashiguchi Y, Muro K, Saito Y, Ito Y, Ajioka Y, Hamaguchi T, et al. Japanese Society for Cancer of the Colon and Rectum (JSCCR) Guidelines 2019 for treatment of colorectal cancer. Int J Clin Oncol. 2020;25:1-42. 
21. Qadeer MA, Rocio Lopez A, Dumot JA, Vargo JJ. Risk factors for hypoxemia during ambulatory gastrointestinal endoscopy in ASA I-II patients. Dig Dis Sci. 2009;54:1035-40.

22. Iber FL, Sutberry M, Gupta R, Kruss D. Evaluation of complications during and after conscious sedation for endoscopy using pulse oximetry. Gastrointest Endosc. 1993;39:620-5.

23. Goudra BG, Singh PM, Penugonda LC, Speck RM, Sinha AC. Jirapinyo $P$, Thompson CC. Sedation challenges: obesity and sleep apnea. Gastrointest Endosc Clin N Am. 2016:26:527-37.

24. Yilmaz M, Aydin A, Karasu Z, Günşar F, Ozütemiz O. Risk factors associated with changes in oxygenation and pulse rate during colonoscopy. Turk J Gastroenterol. 2002;13:203-8.

25. Goudra BG, Singh PM, Penugonda LC, Speck RM, Sinha AC. Significantly reduced hypoxemic events in morbidly obese patients undergoing gastrointestinal endoscopy: Predictors and practice effect. J Anaesthesiol Clin Pharmacol. 2014;30:71-7.

26. Isomoto H, Nishiyama H, Yamaguchi N, Fukuda E, Ishii H, Ikeda K, et al. Clinicopathological factors associated with clinical outcomes of endoscopic submucosal dissection for colorectal epithelial neoplasms. Endoscopy. 2009:41:679-83.

27. Yoshida N, Fernandopulle N, Murakami T, Hirose R, Ogiso K, Inada Y, et al. Difficult manipulation is one of the risk factors for incomplete resection in colorectal endoscopic submucosal dissection. J Gastroenterol Hepatol Res. 2016;5:2070-6.

28. Jain D, Goyal A, Uribe J. Obesity and cecal intubation time. Clin Endosc. 2016:49:187-90.

29. Moon SY, Kim BC, Sohn DK, Han KS, Kim B, Hong CW, et al. Predictors for difficult cecal insertion in colonoscopy: The impact of obesity indices. World J Gastroenterol. 2017;23:2346-54.

30. Akere A, Otegbayo JA. Complete colonoscopy: impact of patients' demographics and anthropometry on caecal intubation time. BMJ Open Gastroenterol. 2016;3:e000076.

31. Ohya T, Ohata K, Sumiyama K, Tsuji Y, Koba I, Matsuhashi N, et al. Balloon overtube-guided colorectal endoscopic submucosal dissection. World J Gastroenterol. 2009;15:6086-90.

32. Yamamoto K, Shimoda R, Ogata S, Hara M, Ito Y, Tominaga N, et al. Perforation and Postoperative Bleeding Associated with Endoscopic Submucosal Dissection in Colorectal Tumors: An Analysis of 398 Lesions Treated in Saga. Japan Intern Med. 2018;57:2115-22.

\section{Publisher's Note}

Springer Nature remains neutral with regard to jurisdictional claims in published maps and institutional affiliations.
Ready to submit your research? Choose BMC and benefit from:

- fast, convenient online submission

- thorough peer review by experienced researchers in your field

- rapid publication on acceptance

- support for research data, including large and complex data types

- gold Open Access which fosters wider collaboration and increased citations

- maximum visibility for your research: over $100 \mathrm{M}$ website views per year

At $\mathrm{BMC}$, research is always in progress.

Learn more biomedcentral.com/submissions 